\title{
A Fast Algorithm of the Geomagnetic Correlation Matching Based on MSD
}

\author{
Weinan Xie, Zhenshen Qu, Qinghua Li \\ Space Control and Inertial Technology Research Center \\ Harbin Institute of Technology \\ Harbin, China \\ xieweinan@hit.edu.cn
}

\begin{abstract}
In this paper, a fast algorithm of the geomagnetic correlation matching based on MSD (mean square difference) is proposed. According to Taylor formula, the expression of MSD is transformed using the gradient of the magnetic field intensity. By introducing the parallelism constraint, the regularization model is established. Linear equations can be obtained with the help of minimizing the regularization model. Then Jacobi iteration is used to obtain the matching result by solving equations. Finally, the results are confirmed by simulation.
\end{abstract} MSD.

Keywords-geomagnetic navigation, geomagnetic matching,

\section{INTRODUCTION}

Geomagnetic navigation is a new passive autonomous navigation method, which has the advantage of all-time, all weather, compact dimension, low weight, no accumulative error and so on. Nevertheless, there are areas where geomagnetic field distribution is very smooth and the database of geomagnetic field information is not complete. If geomagnetic field information is used alone, it is more likely to have precision problem and reliability problem. Inertial navigation system (INS) can provide autonomous and realtime navigation information with good short-term accuracy, which limits geomagnetism navigation range. So geomagnetism aided inertial navigation system can effectively improve system reliability and navigation precision, which has attracted a lot of attention ${ }^{[1,2]}$. With the devolvement of the geomagnetic navigation technology, there are two orientations: geomagnetic filtering navigation and geomagnetic matching navigation.

The principium of geomagnetic filtering navigation is fusing geomagnetic information and other navigation information with filter ${ }^{[3]}$. Geomagnetic field model must be established in the geomagnetic filtering navigation algorithm. So the geomagnetic filtering technology mainly applies to low earth orbit satellite systems, for reducing the impact of the geomagnetic anomalous to the geomagnetic field model. In 90s of last century, Psiaki firstly determined the low-earth orbit using magnetometer data ${ }^{[4,5]}$. A team from Goddard Space Center achieved data fusion of magnetometer information and other sensor information using Kalman filtering technique ${ }^{[6,7]}$. Geomagnetic filtering navigation mainly involves $\mathrm{EKF}^{[1,2]}, \mathrm{UKF}^{[8]}$ and particle filtering ${ }^{[9]}$.
Another way of geomagnetic navigation is geomagnetic matching technology. Geomagnetic matching is the method to find the position which is localized by matching measurements of geomagnetic field to an a priori map stored in computer ${ }^{[10]}$. American E-System Corporation proposed contour-based magnetic matching MAGCOM system in the 1960s. In 1974-1976 the Russian project "MAGNET" was successfully conducted by the Ramenskoye Design Company. The test trajectory corridor that was selected was mapped and computed upward for altitudes up to $8 \mathrm{~km}$ by the Institute of Terrestrial Magnetism ${ }^{[11]}$. In recent years, Chinese scholars began to study geomagnetic matching navigation $^{[12,13]}$.

Based on the study of the correlation matching, a fast matching method using geomagnetic field gradient is developed in this paper. In the computer simulation, good performance is observed.

\section{CORRELATION MATCHING}

Correlation matching is the mainly matching algorithm based on the sequential measure technology and it has been applied successfully in terrain matching area. The classical matching methods include MAD, MSD, PROD, NPROD ${ }^{[14]}$. The correlation functions are given in Table 1 , where $M_{M}^{k}$ is the measured magnetic anomaly, $M_{S}^{k}$ is the magnetic anomaly of the map at position sequence, $N$ is the number of the sample points. Nevertheless, correlation matching needs to go through every trajectory, which is parallel to the reference trajectory, in the matching area. So it is calculation burden for navigation computer and causes a low matching speed.

TABLE I. CORRELATION FUNCTION

\begin{tabular}{|c|c|}
\hline Principles & Formulary $(i=-m, \cdots, m ; j=-n, \cdots, n)$ \\
\hline PROD & $\operatorname{prod}_{i j}=\frac{1}{N} \sum_{k=1}^{N} M_{S(i, j)}^{k} M_{M}^{k}$ \\
\hline NPROD & $\operatorname{nprod}_{i j}=\frac{\sum_{k=1}^{N} M_{S(i, j)}^{k} M_{M}^{k}}{\left[\sum_{k=1}^{N} M_{S(i, j)}^{k}\right]^{1 / 2}\left[\sum_{k=1}^{N} M_{M}^{k}\right]^{1 / 2}}$ \\
\hline MAD & $\operatorname{mad}_{i j}=\frac{1}{N} \sum_{k=1}^{N}\left|M_{S(i, j)}^{k}-M_{M}^{k}\right|$ \\
\hline MSD & $\operatorname{msd}_{i j}=\frac{1}{N} \sum_{k=1}^{N}\left(M_{S(i, j)}^{k}-M_{M}^{k}\right)^{2}$ \\
\hline
\end{tabular}




\section{FAST MSD MATChING PRINCIPLE}

As Fig 1 shows, $C_{r}$ is the reference trajectory and $C_{m}$ is its matching trajectory. Reference trajectory consists of reference point $\left(r_{x i}, r_{y i}\right)^{T}$, and $r_{x i}, r_{y i}$ are longitude and latitude of the reference point $i$, where $i=1,2, \cdots, N, N$ is the total number of points. According to the geomagnetic database, we can get the magnetic field intensity $m\left(r_{x i}, r_{y i}\right)$ of this point, which is a function of the longitude $r_{x i}$ and the latitude $r_{y i} .\left(p_{x i}, p_{y i}\right)^{T}$ is the matching point of the reference point $\left(r_{x i}, r_{y i}\right)^{T}$, and $p_{x i}, p_{y i}$ are longitude and latitude of the matching point. So we can also get the magnetic field intensity $m\left(p_{x i}, p_{y i}\right)$. Then we have

$$
m\left(r_{x i}+e_{x i}, r_{y i}+e_{y i}\right)=m\left(p_{x i}, p_{y i}\right)
$$

where $e_{x i}=p_{x i}-r_{x i}$ is error in longitude from the matching point to the reference point, and $e_{y i}=p_{y i}-r_{y i}$ is error in latitude from the matching point to the reference point.

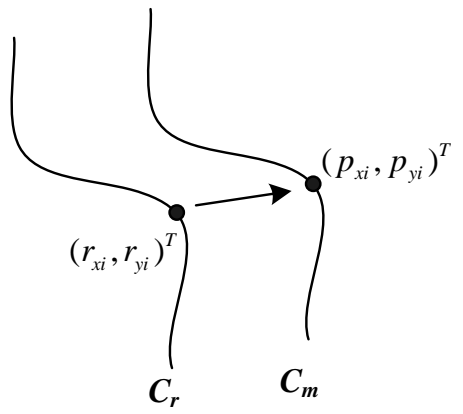

Figure 1. The SCHEMATIC OF THE MATChing PRINCIPLE

The reference point is located nearby the matching point, so we can Taylor expand (1)

$$
m\left(r_{x i}, r_{y}\right)+\frac{\partial m_{i}}{\partial x_{i}} e_{x i}+\frac{\partial m_{i}}{\partial y_{i}} e_{y i}+R_{2 i}=m\left(t_{x i}, t_{y i}\right)
$$

where $\nabla m_{i}=\left[\partial m_{i} / \partial x_{i}, \partial m_{i} / \partial y_{i}\right]$ is the gradient vector of the magnetic field intensity at the reference point $\left(r_{x i}, r_{y i}\right)^{T}$, $R_{2 i}$ is high order expanded term. Neglecting $R_{2 i}$, we have

$$
\nabla m_{i} \cdot \boldsymbol{e}_{\boldsymbol{i}}+\left(m_{i}-l_{i}\right)=0
$$

where $\boldsymbol{e}_{\boldsymbol{i}}=\left[e_{x i}, e_{y i}\right]^{T}$ is the error vector from the matching point to the reference point, $m_{i}=m\left(r_{x i}, r_{y i}\right)$ is the calculated magnetic field intensity of the reference point $\left(r_{x i}, r_{y i}\right)^{T}$ according to the geomagnetic database, and $l_{i}=m\left(t_{x i}, t_{y i}\right)$ is the actual measurement magnetic field intensity of the reference point $\left(r_{x i}, r_{y i}\right)^{T}$.

Furthermore, we suppose that the reference trajectory and the matched trajectory are parallel. So we have the regularization model

$$
E=\sum_{i=1}^{N}\left(c_{i}+\lambda s_{i}\right)
$$

where

$$
\begin{gathered}
c_{i}=\left(\frac{\partial m_{i}}{\partial x_{i}} e_{x i}+\frac{\partial m_{i}}{\partial y_{i}} e_{y i}+\left(m_{i}-l_{i}\right)\right)^{2} \\
S_{i}=\frac{1}{2}\left[\left(e_{x(i+1)}-e_{x i}\right)^{2}+\left(e_{x i}-e_{x(i-1)}\right)^{2}\right. \\
\left.+\left(e_{y(i+1)}-e_{y i}\right)^{2}+\left(e_{y i}-e_{y(i-1)}\right)^{2}\right]
\end{gathered}
$$

and $i=1,2, \cdots, N, N$ is the total number of points, $\lambda>0$ is regulating variable. If $\nabla m_{i}$ and $\left(m_{i}-l_{i}\right)$ can be accurately calculated, we take a larger value of $\lambda$. Otherwise we take a smaller value of $\lambda$. The $\boldsymbol{e}_{\boldsymbol{i}}=\left[e_{x i}, e_{y i}\right]^{T}$, which minimize the regularization model (4), are the solutions.

The first partial derivatives of the regularization model (4) versus $e_{x i}$ and $e_{y i}$ are

$$
\begin{aligned}
& \frac{\partial E}{\partial e_{x i}}=2 \frac{\partial m_{i}}{\partial x_{i}}\left(\frac{\partial m_{i}}{\partial x_{i}} e_{x i}+\frac{\partial m_{i}}{\partial y_{i}} e_{y i}+\left(m_{i}-l_{i}\right)\right)+2 \lambda\left(e_{x i}-\bar{e}_{x i}\right) \\
& \frac{\partial E}{\partial e_{y i}}=2 \frac{\partial m_{i}}{\partial y_{i}}\left(\frac{\partial m_{i}}{\partial x_{i}} e_{x i}+\frac{\partial m_{i}}{\partial y_{i}} e_{y i}+\left(m_{i}-l_{i}\right)\right)+2 \lambda\left(e_{y i}-\bar{e}_{y i}\right)
\end{aligned}
$$

where the average values of errors are

$$
\begin{aligned}
& \bar{e}_{x i}=\frac{1}{2}\left(e_{x(i-1)}+e_{x(i+1)}\right) \\
& \bar{e}_{y i}=\frac{1}{2}\left(e_{y(i-1)}+e_{y(i+1)}\right)
\end{aligned}
$$

The second partial derivatives of the regularization model (4) versus $e_{x i}$ and $e_{y i}$ are

$$
\begin{aligned}
& \frac{\partial^{2} E}{\partial e_{x i}^{2}}=2\left(\frac{\partial m_{i}}{\partial x_{i}}\right)^{2}+2 \lambda \\
& \frac{\partial^{2} E}{\partial e_{y i}^{2}}=2\left(\frac{\partial m_{i}}{\partial y_{i}}\right)^{2}+2 \lambda
\end{aligned}
$$

which are greater than zero. So, $\boldsymbol{e}_{\boldsymbol{i}}=\left[e_{x i}, e_{y i}\right]^{T}$, which make the first partial derivatives of the regularization model (4) versus $e_{x i}$ and $e_{y i}$ equal to zero, are the solutions. Then we have

$$
\begin{aligned}
& \left(\lambda+\left(\frac{\partial m_{i}}{\partial x_{i}}\right)^{2}\right) e_{x i}+\frac{\partial m_{i}}{\partial x_{i}} \frac{\partial m_{i}}{\partial y_{i}} e_{y i}=\lambda \bar{e}_{x i}-\frac{\partial m_{i}}{\partial x_{i}}\left(m_{i}-l_{i}\right) \\
& \frac{\partial m_{i}}{\partial x_{i}} \frac{\partial m_{i}}{\partial y_{i}} e_{x i}+\left(\lambda+\left(\frac{\partial m_{i}}{\partial y_{i}}\right)^{2}\right) e_{y i}=\lambda \bar{e}_{y i}-\frac{\partial m_{i}}{\partial y_{i}}\left(m_{i}-l_{i}\right)
\end{aligned}
$$

where $i=1,2, \cdots, N, N$ is the total number of points.

So it can be solved by Jacobi iteration.

$$
=\bar{e}_{x i}(k)-\frac{\partial m_{i}}{\partial x_{i}} \frac{\frac{\partial m_{i}}{\partial x} \bar{e}_{x i}(k)+\frac{\partial m_{i}}{\partial y} \bar{e}_{y i}(k)+\left(m_{i}-l_{i}\right)}{\lambda+\left(\frac{\partial m_{i}}{\partial x_{i}}\right)^{2}+\left(\frac{\partial m_{i}}{\partial y_{i}}\right)^{2}}
$$




$$
\begin{aligned}
& e_{y i}(k+1) \\
= & \left.\bar{e}_{y i}(k)-\frac{\partial m_{i}}{\partial y_{i}} \frac{\frac{\partial m_{i}}{\partial x} \bar{e}_{x i}(k)+\frac{\partial m_{i}}{\partial y} \bar{e}_{y i}(k)+\left(m_{i}-l_{i}\right)}{\lambda+\left(\frac{\partial m_{i}}{\partial x_{i}}\right)^{2}+\left(\frac{\partial m_{i}}{\partial y_{i}}\right)^{2}}(16)\right)
\end{aligned}
$$

where $k$ is the number of iterations, $e_{x i}(0)$ and $e_{y i}(0)$ are the initial values, which can be set to zero.

Then the matching results are

$$
\begin{aligned}
& p_{x i}=e_{x i}+r_{x i} \\
& p_{y i}=e_{y i}+r_{y i}
\end{aligned}
$$

According to formula (2), we know that we neglect the high order expanded term $R_{2}$. So the matching error becomes greater, as the initial position error increases. Then we can take a few of iteration to enhance matching accuracy. That is, we regard the first matching result as the next reference trajectory.

\section{FASt MSD Matching PRocess}

In practical application, the fast MSD matching process only needs to solve the Jacobi iteration. And the main steps of the algorithm are depicted as follows:

Step 1: For every reference point $\left(r_{x i}, r_{y i}\right)^{T}$ along the trajectory, find the point magnetic field intensity $m\left(r_{x i}, r_{y i}\right)$ and its gradient $\nabla m_{i}$ in the database;

Step 2: Compute the average values of errors $\bar{e}_{x i}(k)$ and $\bar{e}_{y i}(k)$ ( $k$ denotes the $k t h$ iteration) according to (9) and (10);

Step 3: Compute the errors $e_{x i}(k+1)$ and $e_{y i}(k+1)$ according to (15) and (16);

Step 4: Make sure whether the formula (19) is true, where $\varepsilon \geq 0$ is a given small positive number. If it does, iteration is stopped, then (17) and (18) are the matching results; otherwise go on to carry out Step 2.

$$
\frac{1}{N} \sum_{i=1}^{N}\left(\left(e_{x i}(k+1)-e_{x i}(k)\right)^{2}+\left(e_{y i}(k+1)-e_{y i}(k)\right)^{2}\right)<\varepsilon
$$

\section{Simulation Results}

In this section, the simulation is hold to test the validity of the proposed algorithm. The geomagnetic grid form data of some Europe area is applied for simulation experiments, which has spacing about $0.001^{\circ}$. The initial position errors of INS are $-0.01^{\circ}$ in longitude and $0.01^{\circ}$ in latitude. Then the matching result is shown in fig. 2 regardless of the magnetic sensor noise. The average matching errors are $0.0008^{\circ}$ in longitude and $0.0004^{\circ}$ in latitude. The matching time is less than $7 \mathrm{~ms}$.

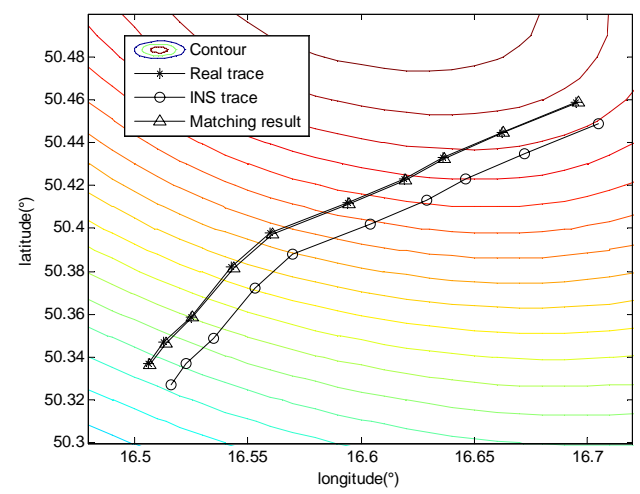

Figure 2. Matching Result Regardless of the Magnetic Sensor NOISE

If the geomagnetic signal contains random noise with amplitude value of $0.5 \mathrm{nT}$, the matching results is shown in fig. 3 with same conditions. The average matching errors are $0.0010^{\circ}$ in longitude and $0.0012^{\circ}$ in latitude. The matching time is less than $11 \mathrm{~ms}$.

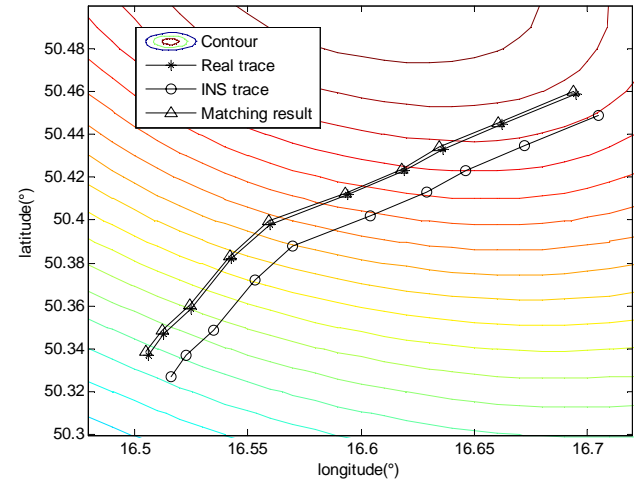

Figure 3. MATCHING RESUlt WITH RANDOM NOISE

When the initial error is increased, we can call iteration algorithms repeatedly. The initial position errors of INS are $-0.1^{\circ}$ in longitude and $0.1^{\circ}$ in latitude. Then we repeat the process three times, and the matching result is shown in fig. 4 regardless of the magnetic sensor noise. The average matching errors are $0.0011^{\circ}$ in longitude and $0.0004^{\circ}$ in latitude. The matching time is less than $17 \mathrm{~ms}$.

If the geomagnetic signal contains random noise with amplitude value of $0.5 \mathrm{nT}$, the matching results is shown in fig. 5 with same conditions. The average matching errors are $0.0001^{\circ}$ in longitude and $0.0012^{\circ}$ in latitude. The matching time is less than $18 \mathrm{~ms}$.

The simulation shows that the fast MSD matching can obtain accurate result in less computational time. 


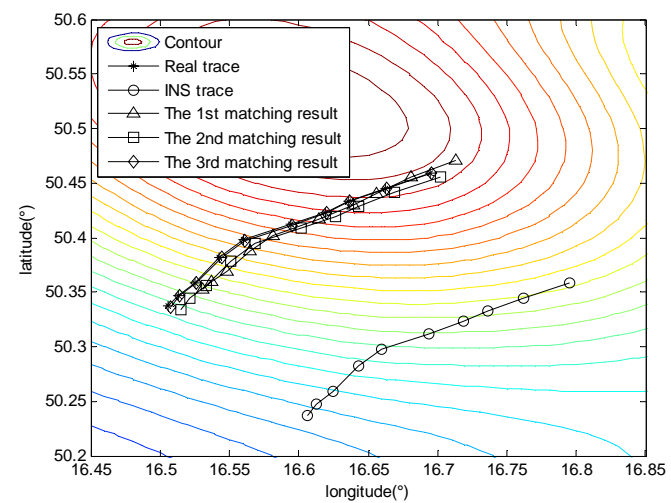

Figure 4. MAtChing Result RegardLESS of the Magnetic SENSOR NOISE

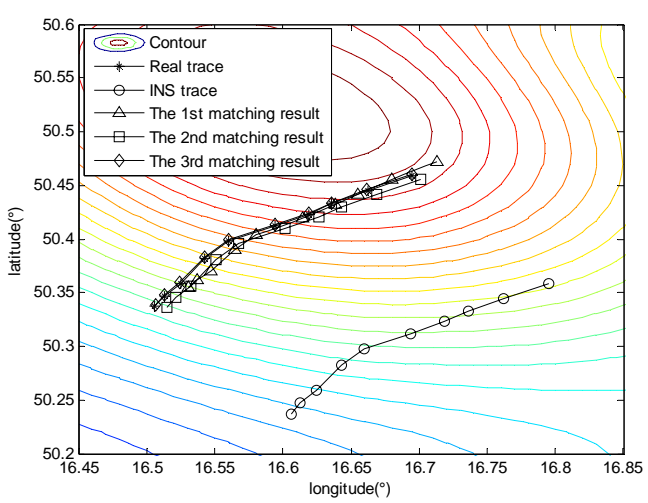

Figure 5. MATCHING RESUlt WITH RANDOM NOISE

\section{CONCLUSIONS}

Real-time of geomagnetic correlation matching is constrained by the research scope and the research step length. Then, in this paper a fast iterative algorithm for geomagnetic correlation matching is proposed. The matching perform indicator is converted into the discrete regularization model. Taking the derivative of this model, linear equations are established. With Jacobi iteration, the main steps of the fast matching algorithm are given. At last, the performance of the algorithm is verified for different initial error. The next issue for future work is to obtain matching process with initial heading angle error between the reference trajectory and the matching trajectory.

\section{REFERENCES}

[1] M. Barczyk, A. F. Lynch, "Integration of a triaxial magnetometer into a helicopter UAV GPS-aided INS,” IEEE Transaction on Aerospace and Electronic Systems. Vol. 48, pp. 2947-2960, 2012.

[2] H. Ren, P. Kazanzides, "Investigation of attitude tracking using an integrated inertial and magnetic navigation system for hand-held surgical instruments," IEEE/ASME Transactions on Mechatronics, vol. 17, pp. 210-217, Sep. 2012.

[3] Z. Guo, L. Luan, H. Zhu, et al, "Geomagnetic filtering navigation algorithm based on single spot matching precorrection,” Proceedings of the Fifth International Symposium on Computational Intelligence and Design, pp. 543-546, 2012.

[4] M. L. Psiaki, L. Huang, S. M. Fox, "Ground tests of magnetometerbased autonomous navigation (MAGNAV) for low-earth-orbiting spacecraft,” Journal of Guidance, Control, and Dynamics, vol. 16, pp. 206-214, 1993.

[5] M. L. Psiaki, “Autonomous low-earth orbit determination from magnetometer and star sensor data," Journal of Guidance, Control, and Dynamics, vol. 22, pp. 296-304, 1999.

[6] J. Deutschmann, I. Y. Bar-Itzhack, "Evaluation of attitude and orbit estimation using actual earth magnetic field data," Journal of Guidance, Control, and Dynamics, vol. 24, pp. 616-626, 2001.

[7] J. K. Thienel, R. R. Harman, I. Y. Bar-Itzhack, et al, "Results of the Magnetometer navigation (MAGNAV) in flight experiment," AIAA/AAS Astrodynamics Specialist Conference, pp. 206-222, 2004.

[8] G. Yang, S. Li, Z Jiang, "Data fusing algorithm in geomagnetic aided INS,” Journal of Chinese Inertial Technology, vol. 15, pp. 47-51, 2007.

[9] W. Kwon, K. S. Roh, H. K. Sung, "Particle filter-based heading estimation using magnetic compasses for mobile robot navigation," Proceedings of IEEE International Conference on Robotics and Automation, pp. 2705-2712, 2006.

[10] Z. Ren, L, Chen, H. Zhuang, et al, "Research on geomagneticmatching localization algorithm for unmanned underwater vehicles," Proceeding of IEEE International Conference on Information and Automation, pp. 1025-1029, 2008.

[11] F. Goldenberg, "Geomagnetic navigation beyond the magnetic compass,” Proceeding of IEEE Position, Location, and Navigation Symposium, pp. 2705-2712, 2006.

[12] S. Luo, Y. Wang, Y. Liu, et al, "Research on Geomagnetic-matching technology based improved ICP algorithm,” Proceedings of IEEE International Conference on Information and Automation, pp. 815819, 2008.

[13] W. Liu, Z. Wu, M. Wu, et al, "Geomagnetic matching technology based on iterative contour matching algorithm," Proceedings of the Tenth International Conference on Electronic Measurement \& Instruments, pp. 342-345, 2011.

[14] Y. Liu, M. Wu, X. Hu, et al, "Research on geomagnetic matching method," Proceedings of the second International Conference on Industrial Electronics and Applications, pp. 2701-2711, 2007. 